\title{
Regulation of Autophagy by High Glucose in Human Retinal Pigment Epithelium
}

\author{
Jin Yao a,b Zhi-Fu Tao a,b Chao-Peng Lia Xiu-Miao Lia Guo-Fan Cao ${ }^{a}$ Qin Jianga \\ Biao Yan ${ }^{a}$
}

aEye Hospital, Nanjing Medical University, Nanjing, China; 'bThese authors contributed equally to this work

\author{
Key Words \\ High glucose $\bullet$ Autophagy $\bullet$ ROS $\bullet$ ER stress
}

\begin{abstract}
Background: Autophagy is a self-degradative process that is important for balancing sources of energy at critical times in development and in response to nutrient stress. Retinal pigment epithelium (RPE) works as the outer blood retina barrier and is vulnerable to energy stressinduced injury. However, the effect of high glucose treatment on autophagy is still unclear in RPE. Methods: Transmission electron microscopy was used to detect the generation of autophagosome. Small interfering RNA (siRNA) and MTT was used to determine the effect of autophagy on cell viability. Western blots and immunohistochemistry were used to detect the expression pattern of autophagic markers, including LC3 and p62. Results: High glucose treatment results in a significant increase in the generation of autophagosome and altered expression of LC3 and p62. High glucose-induced autophagy is independent of mTOR signaling, but is mainly regulated via ROS-mediated ER stress signaling. Conclusion: In the scenario of high glucose-induced oxidative stress, autophagy may be required for the removal of damaged proteins, and provide a default mechanism to prevent high glucose-induced injury in RPE.
\end{abstract}

Copyright (C) 2014 S. Karger AG, Basel

\section{Introduction}

Autophagy refers as a catabolic process that involves the degradation of cellular components through lysosomal machinery. It helps to maintain the balance between the synthesis, degradation, and subsequent recycling of cellular components [1]. Mounting evidence suggests that autophagy plays an important role in cell growth, development, 


\section{Cellular Physiology and Biochemistry}

Cell Physiol Biochem 2014;33:107-116

\begin{tabular}{l|l}
\hline DOI: $10.1159 / 000356654$ & (C) 2014 S. Karger AG, Basel
\end{tabular}

\begin{tabular}{l|l} 
Published online: January 17, 2014 & www.karger.com/cpb
\end{tabular}

Yao et al.: High Glucose Induces RPE Autophagy

and homeostasis [2]. Disruption of autophagic process results in the failure of cavitation during embryogenesis or the accumulation of abnormal mitochondria in adult tissues $[3,4]$. Moreover, malfunction of autophagy has been linked to a wide range of human pathologies, including cancer, neurodegeneration, and pathogen infection [5, 6]. Thus, autophagy should be carefully regulated and executed, which is important for the maintenance of cellular homeostasis as well as being a crucial adaptation mechanism against a multitude of cellular stress conditions.

Diabetic retinopathy is one of the most serious diabetic complications, which could cause the irreversible blindness $[7,8]$. The retinal vasculature is particularly vulnerable to be damaged in patients with diabetes. Nearly all types of retinal cells are at risk. Notable among these cells are retinal pigment epithelium (RPE), which performs a number of vital functions to maintain the overall health of the retina, such as transport of nutrients and removal of waste products from photoreceptor cells, retinoid transport and regeneration, and immune/ inflammation $[9,10]$. RPE is located between the photoreceptors and the choriocapillaries, which constitutes the outer blood-retina barrier. Disruption of the blood-retina barrier not only disturbs normal fluid flow between the neural retina and the choriocapillaries, resulting in edemas, but also affects the metabolic circuits and RPE function [11]. Thus, it is required to maintain normal physiological and functional properties of RPE during diabetic retinopathy.

Hyperglycemia plays an important role in the pathogenesis of diabetic complications. The toxic effects of hyperglycemia have been corroborated in numerous in vitro and in vivo studies [12,13]. Autophagy can function as a survival mechanism that allows the cells to maintain energy homeostasis and viability. It is also is important for the removal of damaged proteins and organelles. Autophagy thereby confers stress tolerance, limits damage, and sustains viability under adverse conditions $[1,2]$. However, the functional role of autophagy in RPE is still unclear upon high glucose stress. In this study, we found that high levels of glucose could induce autophagy in RPE. This induction of autophagy is independent of mTOR signaling, but is mainly mediated by ROS-mediated ER stress signaling.

\section{Materials and Methods}

\section{Cell culture}

Human RPE cell line, ARPE-19, was obtained from the American Type Culture Collection (Manassas, VA). It was cultured in Dulbecco's modified eagle medium (DMEM) medium and maintained at $37^{\circ} \mathrm{C}$ in a humidified chamber of $5 \% \mathrm{CO}_{2}$. The culture medium was replaced with fresh medium every other day.

\section{Transmission electron microscopy}

RPE were fixed in $2.5 \%$ glutaradehyde in $0.1 \mathrm{M}$ sodium cacodylate buffer ( $\mathrm{pH} 7.0$ ) for 1 $\mathrm{h}$, fixed in $1 \%$ osmium tetroxide in $0.1 \mathrm{M}$ cacodylate buffer for $1 \mathrm{~h}$, dehydrated with increased concentrations of ethanol, and gradually infiltrated with araldite resin. Ultrathin sections (70-80 $\mathrm{nm})$ were obtained using an ultramicrotome. Sections were stained with uranyl acetate and lead citrate, and then examined using a Hitachi H-7500.

\section{Immunohistochemistry (IHC) assay}

RPE were fixed in 4\% paraformaldehyde for $15 \mathrm{~min}$. Following fixation, these cells were permeabilized with $0.2 \%$ Triton X-100, blocked with $10 \%$ BSA solution. They were incubated with LC3 antibody for overnight at $4^{\circ} \mathrm{C}$ followed by $1 \mathrm{~h}$ incubation of the secondary antibody conjugated with Alexa fluor 568 (1:200 dilution). These samples were observed using a fluorescence microscopy (Leica).

Determination of reactive oxygen species (ROS)

The concentration of ROS in RPE was determined by measuring the fluorescent signal from the redox-sensitive fluoroprobe, 2', 7'-dichlorofluorescein diacetate (DCFDA). Briefly, 


\section{Cellular Physiology and Biochemistry}

Cell Physiol Biochem 2014;33:107-116

\begin{tabular}{l|l}
\hline DOI: $10.1159 / 000356654$ & (C) 2014 S. Karger AG, Basel
\end{tabular}

\begin{tabular}{l|l} 
Published onlıne: January 17, 2014 & www.karger.com/cpb
\end{tabular}

Yao et al.: High Glucose Induces RPE Autophagy

RPE were grown in 6-well plates in phenol red-free DMEM, and then incubated with normal glucose $(5 \mathrm{mM})$ or high glucose $(30 \mathrm{mM})$ for the indicated times. These cells were washed in phosphate-buffered saline (PBS buffer), and DCFDA (10 $\mathrm{mM})$ in serum-free medium was added for $40 \mathrm{~min}$ at $37^{\circ} \mathrm{C}$. DCF fluorescence in the supernatant was measured with an excitation wavelength of $485 \mathrm{~nm}$ and an emission wavelength of $525 \mathrm{~nm}$.

\section{Western blot}

The treated cells were washed three times with ice-cold PBS, homogenized in the lysis buffer. Total protein concentration was measured using the BCA Protein Assay Kit (Thermo) with bovine serum albumin (BSA) as a standard. Individual samples containing $50 \mu \mathrm{g}$ total proteins were run on the SDS-polyacrylamide gel, and then transferred to PVDF membrane. The membrane was blocked for $1 \mathrm{~h}$, and then the primary antibody was incubated with the membrane overnight at $4^{\circ} \mathrm{C}$. The membrane was washed and incubated with the secondary antibody labeled with horseradish peroxidase for $1 \mathrm{~h}$ at room temperature. The bands were visualized using the enhanced chemiluminescence (ECL) method.

\section{Detection of cell viability}

The 3-(4,5-dimethylthiazal-2-yl)-2,5-diphenyl-tetrazolium bromide (MTT) assay was used to detect cell viability. Briefly, ARPE-19 cells were plated onto 96-well plates in 100 $\mu \mathrm{l}$ growth medium and allowed to adhere overnight. These cells were transfected with or without $50 \mathrm{nM}$ ATG5 siRNA or scrambled siRNA using lipofectamine 2000 (Invitrogen), and then treated as shown in figure legend. After the removal of the medium, $100 \mathrm{mM}$ DMSO solution was added to dissolve the formazan crystals. The absorbance at $570 \mathrm{~nm}$ wave length was detected using a microplate reader (Thermo).

\section{Statistical Analysis}

All data were presented as mean \pm S.E.M.. Student's $t$ test and one-way ANOVA with post hoc Tukey test were employed for two-group and multiple comparisons, respectively. $P$ values less than 0.05 were considered to represent statistically significant differences.

\section{Result}

\section{High glucose induces RPE autophagy}

To determine the effect of high glucose on autophagy, RPE were incubated in the medium containing normal glucose $(5 \mathrm{mM})$ and high glucose $(30 \mathrm{mM})$, respectively. Transmission electron microscopy observation showed that high levels of glucose resulted in a significant increase in the number of double-membrane vacuoles, which is typical of autophagosomes (Fig. 1A). Autophagy is a multifaceted process and has paradoxically been reported to have roles in promoting both cell survival and cell death [14]. We employed MTT method to investigate the potential role of autophagy in RPE viability upon high glucose stress. We found that compared with the control group, high glucose treatment did not significantly alter cell viability of wild-type RPE or Scr siRNA-transfected RPE. By contrast, high glucose resulted in an obvious reduction in cell viability of autophagy-deficient RPE (ATG5 siRNA transfection) (Fig. 1B), suggesting that autophagy plays a protective role in RPE upon high glucose stress.

\section{High glucose alters the expression pattern of autophagic markers}

In addition to electron microscopy observation, light microscopy detection of the subcellular localization of LC3, and biochemical detection of the membrane-associated form of LC3 are two important methods to monitor the number of autophagosomes [15, 16]. Light microscopy detection demonstrated that LC3 expression was evenly distributed within RPE under normal physiological condition. High glucose treatment altered the distribution of LC3 protein from the diffused staining to punctate staining, typical of autophagosome (Fig. 


\section{Cellular Physiology and Biochemistry}

Cell Physiol Biochem 2014;33:107-116

\begin{tabular}{l|l}
\hline DOI: $10.1159 / 000356654$ & (C) 2014 S. Karger AG, Basel
\end{tabular}

Published online: January 17, $2014 \quad$ www.karger.com/cpb

Yao et al.: High Glucose Induces RPE Autophagy
Fig. 1. High glucose treatment induces RPE autophagy. (A) RPE were incubated in the medium containing normal glucose $(5 \mathrm{mM})$ or high glucose $(30 \mathrm{mM})$ for $48 \mathrm{~h}$, and then these cells were prepared for transmission electron microscopy observation. A representative electron microphotograph of a cell treated with normal glucose or high glucose along with the statistical data (the average number of autophagosome in each cell) is shown. Arrow indicates autophagosome in RPE, and “*” indicates a significant difference compared with the control group; (B) RPE were transfected with ATG5 siRNA, scramble siRNA (Scr siRNA), or left untreated, and then these cells were treated with as shown in Fig. 1A. The cell viability was determined using MTT assay. Results are expressed mean \pm S.E.M. of four independent experiments. "*" indicates a significant difference compared with the control group.

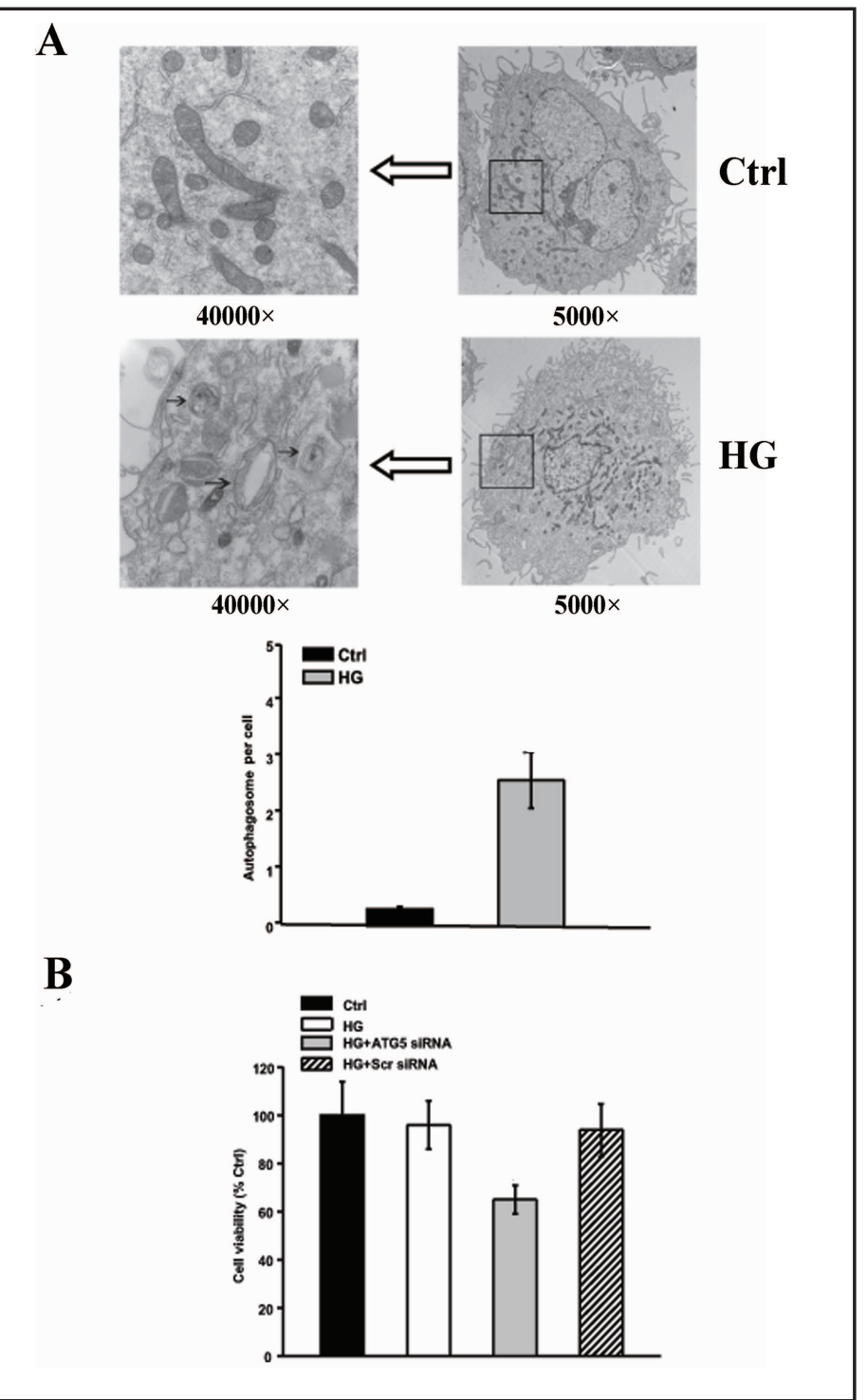

2A). Once autophagy is initiated, LC3 is processed from LC3-I (16 kDa) to LC3-II (14 kDa), and then incorporated into autophagic vacuoles. Thus, the percentage of LC3 conversion can be used to reflect the number of autophaosome $[15,16]$. As shown in Fig. 2B, western blot revealed that high glucose resulted in a marked increase in LC3-II conservation, suggesting that high levels of glucose induces RPE autophagy.

The number of autophagosomes observed at any specific time point is a function of balance between the rate of their generation and the rate of their degradation inside the lysosome. Autophagic flux can be measured by inferring LC3-II turnover by western blot in the presence and absence of lysosomal degradation $[15,16]$. Here, we found that high glucose resulted in a significant increase in LC3-II turnover. $\mathrm{NH}_{4} \mathrm{Cl}$, a lysosomal inhibitor, could interrupt the fusion of lysosome-autophagosome fusion. $\mathrm{NH}_{4} \mathrm{Cl}$ treatment could further increase LC3-II amount (Fig. 2B). The difference of LC3-II amount in the presence and absence of lysosomal inhibitor represent the amount of LC-3 that is delivered to lysosomes for degradation. Further, we found that LC3-II amount under high glucose condition (with or without $\mathrm{NH}_{4} \mathrm{Cl}$ ) was greater than that in normal condition, suggesting autophagic flux is increased upon high glucose stress.

QSTM1/p62 is selectively incorporated into autophagosomes through the direct binding to LC3 and is efficiently degraded by autophagy. Thus, p62 amount inversely correlates with 


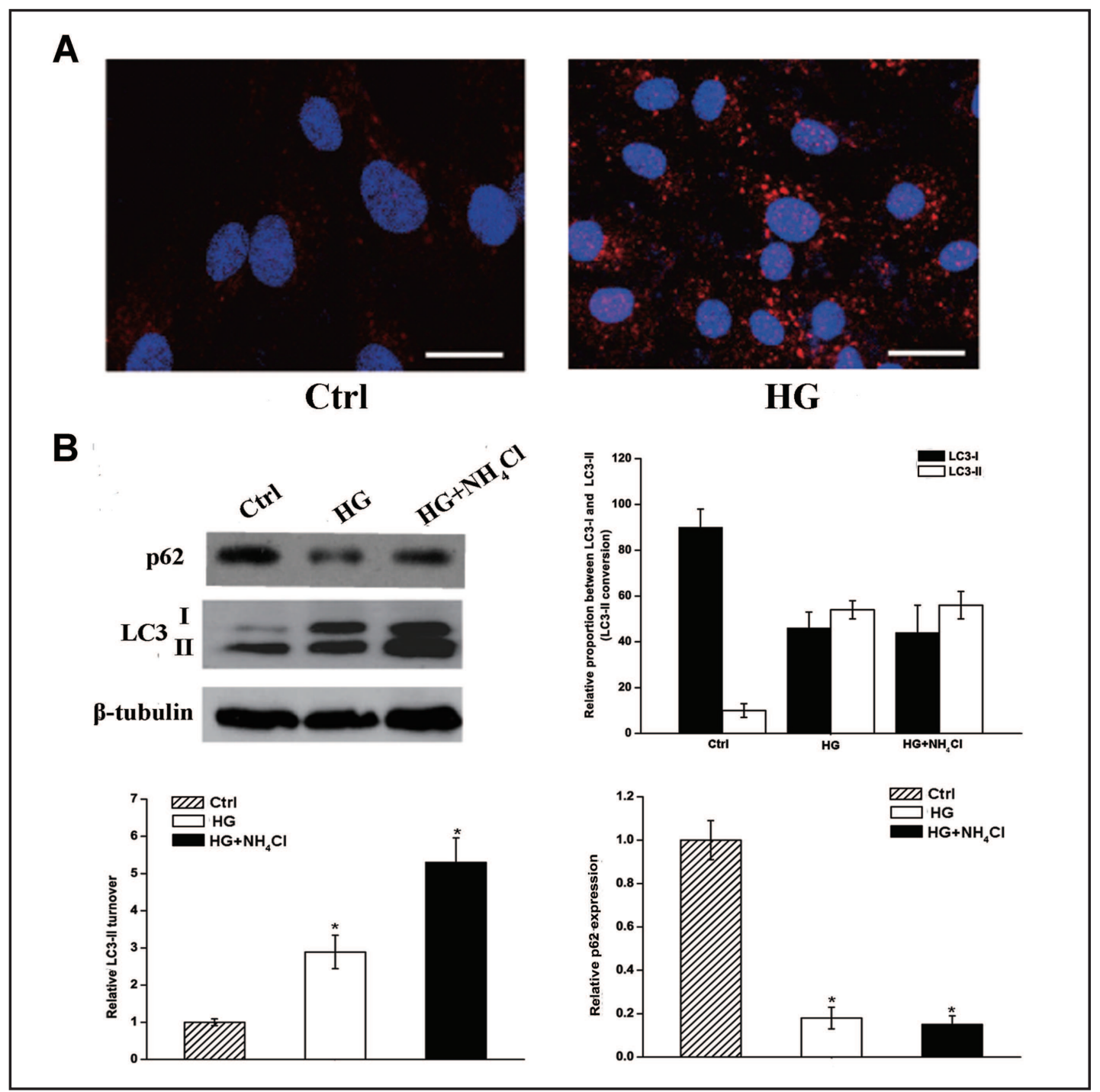

Fig. 2. High glucose alters the expression pattern of autophagic markers. (A) RPE were incubated in medium containing normal glucose ( $5 \mathrm{mM})$ or high glucose $(30 \mathrm{mM})$ for $48 \mathrm{~h}$. These cells were fixed, and immunolabeled with LC3 antibody (Cell Signaling, Product No: \#2775, 1:200) followed by Alexa fluor 568-conjugated secondary antibody. Finally, these cells were stained with DAPI to visualize the nuclei (blue). A representative image is shown. Scale bars $=10 \mu \mathrm{m}$. (B) RPE were incubated in the medium containing normal glucose $(5 \mathrm{mM})$, high glucose $(30 \mathrm{mM})$ with or without $\mathrm{NH}_{4} \mathrm{Cl}$. Western blots were conducted to determine the level of LC3 or p62 expression. $\beta$-tubulin expression was detected as the loading control. The group treated with normal glucose was taken as the control group. The relative amount of LC3-II conversion, LC3-II turnover, and p62 was expressed as the fold increase compared with the control group. A representative immunoblot is shown along with the quantitative data showing the mean \pm S.E.M. from four separate blots.

autophagic activity [15]. We found that compared with LC3-II expression, p62 protein has an opposite expression trend (Fig. 2B). Taken together, the expression data of LC3-II and p62 indicates that high glucose treatment could induce autophagy in RPE.

High glucose-induced autophagy is independent of mTOR signaling pathway

The process of autophagy is regulated by external nutritional status. The mammalian target of rapamycin (mTOR) is a sensor of cellular nutritional status, which has been reported to play a critical role in autophagy [17]. To investigate the association between mTOR 
Fig. 3. High glucosemediated autophagy is independent of mTOR signaling. (A) RPE were incubated in medium containing normal glucose (5 mM) or high glucose (30 mM) for $48 \mathrm{~h}$. The group treated with normal glucose was taken as the control group. Western blots were conducted to determine the phosphorylation levels of S6K and 4E-BP1 expression. $\beta$-tubulin expression was detected as the loading control. The relative amount of S6K or 4E-BP1 was expressed as the fold increase compared with the control group. A representative immunoblot is shown along with the quantitative data showing the mean \pm S.E.M. from four separate blots. (B) RPE were incubated in medium containing normal glucose $(5 \mathrm{mM})$ or high glucose (30 mM) with or without rapamycin (Rap) for $48 \mathrm{~h}$. The

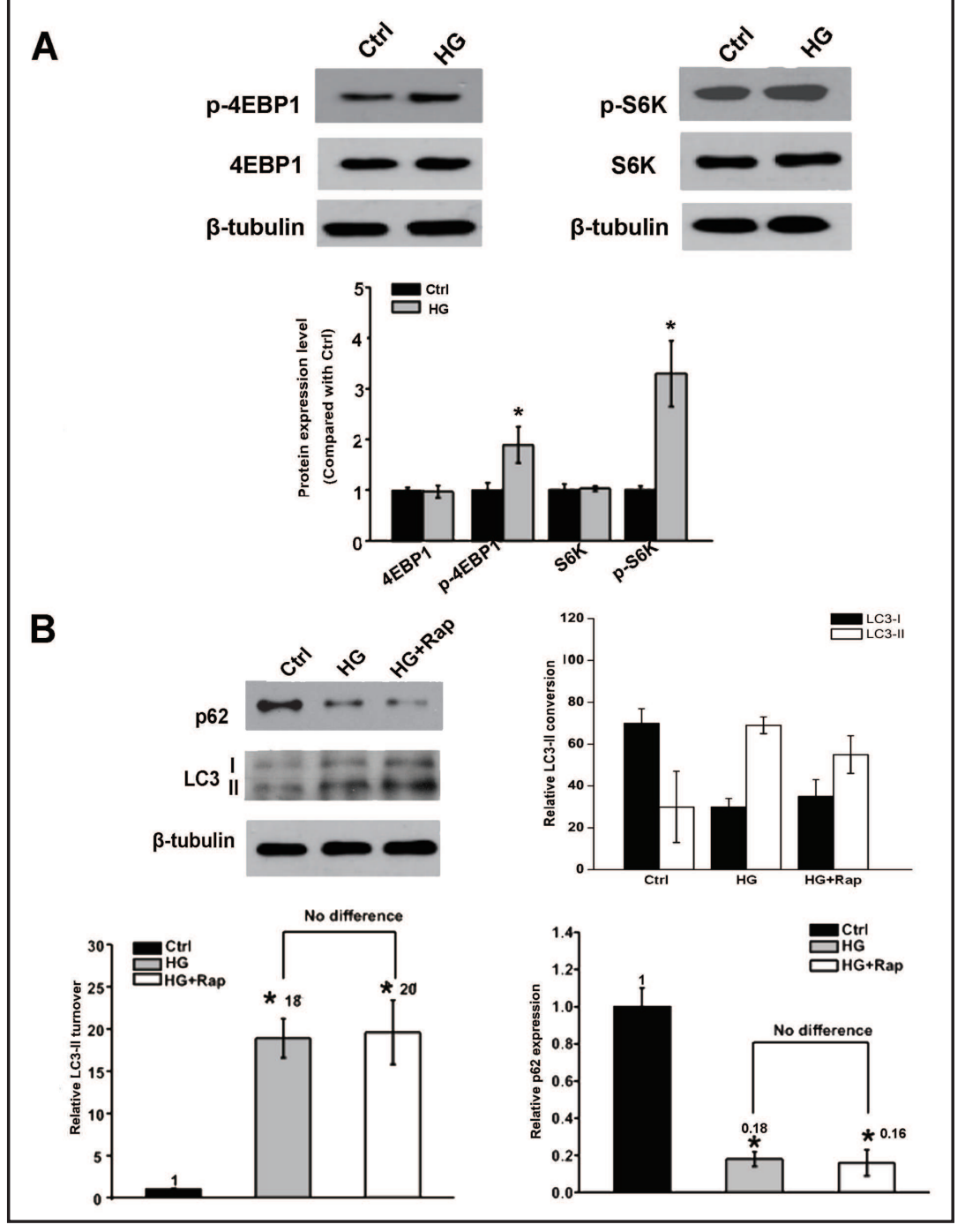

group treated with normal glucose was taken as the control group. Western blots were conducted to determine the level of LC3 or p62 expression. $\beta$-tubulin expression was detected as the loading control. A representative immunoblot is shown along with the quantitative data showing the mean \pm S.E.M. from four separate blots.

activation and autophagy induction, we detected the expression pattern of two downstream effectors of mTOR signaling, S6K and 4EBP1, in response to high glucose stress. High glucose did not affect the total amount of S6K and 4EBP1, but resulted in a significant increase in the levels of phosphorylated S6K and 4EBP1 (Fig. 3A), suggesting that high levels of glucose could activate mTOR signaling. To determine the direct role of mTOR signaling in autophagy, RPE were treated with or without rapamycin to regulate mTOR activity. The result showed that compared with high glucose-treated group, the inhibition of mTOR signaling had no effect on high glucose-induced LC3-II conversion, LC3-II turnover and p62 down-regulation (Fig. 3B), suggesting mTOR is not the major signaling pathway involved in the regulation of high glucose-induced autophagy.

High glucose induces autophagy through ROS-mediated ER stress signaling pathway

Nutrient excess could lead to an obvious increase in ROS gneration, which is a potent regulator of MAP kinase family members, such as c-Jun amino-terminal kinase (JNK) [18]. 


\section{Cellular Physiology and Biochemistry}

Cell Physiol Biochem 2014;33:107-116

\begin{tabular}{l|l}
\hline DOI: 10.1159/000356654 & (c) 2014 S. Karger AG, Basel
\end{tabular}

\begin{tabular}{l|l} 
Published online: January 17, 2014 & www.karger.com/cpb
\end{tabular}

Yao et al.: High Glucose Induces RPE Autophagy

Fig. 4. High glucose induces autophagy through ROS-mediated ER stress signaling. (A) RPE were incubated in the medium containing the different concentrations of glucose (5 mM, $20 \mathrm{mM}, 25 \mathrm{mM}$, and $30 \mathrm{mM}$ ) for $48 \mathrm{~h}$ or incubated in high glucose (30 mM) for $12 \mathrm{~h}, 24 \mathrm{~h}$, or $48 \mathrm{~h}$. Intracellular ROS production was detected by using DCFDA dye. The group treated with normal glucose was taken as the control group. The data was expressed as the relative fold increase compared with the control group. "* " indicates significant difference compared with the control group. (B) RPE were incubated in the medium containing normal glucose (5 $\mathrm{mM})$, high glucose $(30 \mathrm{mM})$, or mannitol (30 mM) for 48 h. The group treated with normal glucose was taken as the control group. Western blots were conducted to determine the total amount and the phosphorylation level of JNK protein. $\beta$-tubulin expression was detected as the loa-
A

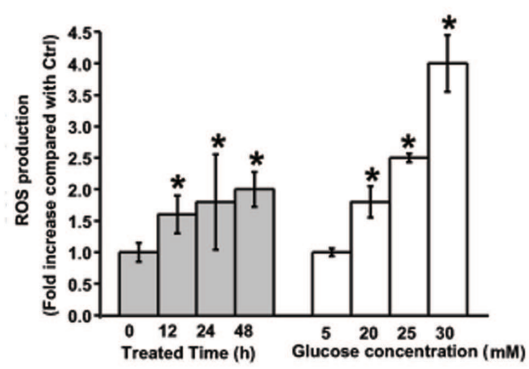

C

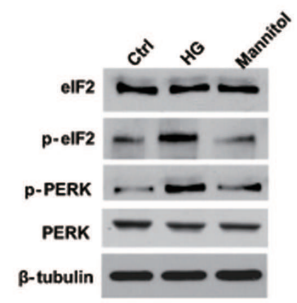

D
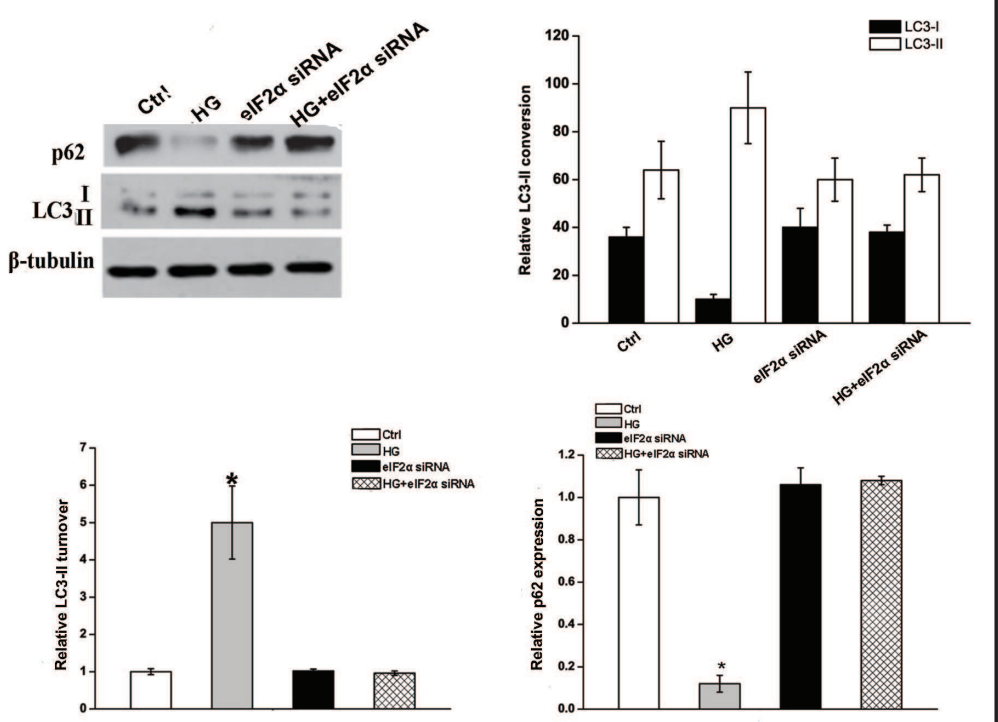

\section{B}

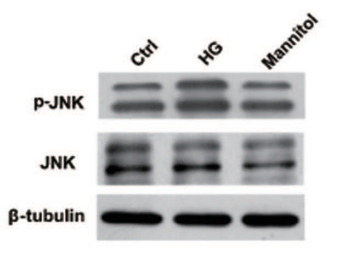

ding control. A representative immunoblot is shown. (C) RPE were treated as shown Fig.4B. Western blots were conducted to determine the levels of p-eIF2, eIF2, PERK, and p-PERK expression. The relative amount of each gene was expressed as the relative change compared with the control group. A representative immunoblot is shown along with the quantitative data showing the mean \pm S.E.M. from four separate blots. (D) RPE were transfected eIF-2 $\alpha$ siRNA to silence ER stress signaling, and then incubated in the medium containing normal glucose $(5 \mathrm{mM})$ or high glucose $(30 \mathrm{mM})$ for $48 \mathrm{~h}$. Western blots were conducted to determine the levels of LC3 and p62 expression. $\beta$-tubulin expression was detected as the loading control. The group treated with normal glucose was taken as the control group. LC3 or p62 expression was expressed as the relative change compared with the control group. A representative immunoblot is shown along with the quantitative data showing the mean \pm S.E.M. from four separate blots.

Here, we found that high glucose could induce ROS generation in a time- and concentrationdependant manner in RPE (Fig. 4A). High glucose did not affect the total amount of JNK, but resulted in a significant increase in the levels of phosphorylated JNK (Fig. 4B). 


\section{Cellular Physiology and Biochemistry}

Cell Physiol Biochem 2014;33:107-116

\begin{tabular}{l|l}
\hline DOI: $10.1159 / 000356654$ & (c) 2014 S. Karger AG, Basel
\end{tabular}

\begin{tabular}{l|l} 
Published online: January 17, 2014 & www.karger.com/cpb
\end{tabular}

Yao et al.: High Glucose Induces RPE Autophagy

Previous study reported that excess ROS results in post-translational modifications of proteins and protein aggregation, which induces endoplasmic reticulum (ER) stress, thereby leads to autophagy induction [18]. The first response to ER stress is transient global translation attenuation mediated by the PKR-like ER kinase (PERK), and then the PERK kinase phosphorylates eukaryotic translation initiation factor $2 \alpha$ (eIF2 $\alpha$ ), which results in translation attenuation [19]. We found that high glucose did not affect the total amount of eIF2 $\alpha$ or PERK expression, but resulted in a marked increase in the phosphorylation level of eIF2 $\alpha$ or PERK (Fig. 4C), suggesting that high glucose treatment could activate ER stress signaling. To determine the direct role of ER stress signaling in autophagy, we tranfected eIF2 $\alpha$ siRNA into RPE to interrupt ER stress signaling. The result showed that high glucose treatment resulted in a obvious increase in LC3-II turnover and conversion, and a reduction in p62 expression, while eIF $2 \alpha$ siRNA transfection could eliminate these effects induced by high glucose (Fig.4D), indicating that high glucose-induced autophagy is mainly regulated through ER stress signaling.

\section{Discussion}

In the eye, the blood retinal barrier (BRB) is a physiological barrier, which could protect the neural retina from molecules and cells in the blood. The inner BRB is formed by normal retinal vessels, while the outer BRB is primarily comprised of the RPE. The healthy RPE not only is critical for the integrity of BRB but also keeps a balanced outer retinal environment $[10,20]$. Hyperglycemia is responsible for the initiation and progression of diabetic retinopathy [7]. In the present study, we demonstrated that high glucose could directly induce autophagic flux in RPE, which appears to be a protective role against high glucoseinduced RPE injury. Specifically, the suppression of autophagy by ATG5 siRNA tranfection could result in a marked reduction in RPE cell viability upon high glucose stress.

Autophagy is an evolutionarily conserved mechanism for the degradation of cellular components in the cytoplasm. It is reported to be a double-edged sword for cell physiology $[1,2]$. It can serve as a cell survival mechanism during the condition of nutrient stress, and can also play a critical role in cell death [21]. Previous studies have revealed that high levels of glucose could promote autophagy in podocyte through the inhibition of mTOR signaling [22]. In pancreatic $\beta$-cells, autophagy could protect against diabetes-induced oxidative injury. We provided a direct evidence that autophagy plays a protective role in RPE [23]. By contrast, Kobayashi et al. found an opposite phenomemon that the suppression of autophagy is protective in high glucose-induced cardiomyocyte injury [24]. Taken together, these results underscore a fact that autophagy could be either protective or detrimental depending on the cell type and cellular environment. Thus, the functional significance of autophagy should be individually determined under different pathological conditions.

RPE are responsible for the exchange of nutrients and metabolites between retina and choriocapillaris, which are particularly susceptible to oxidative injury $[9,10]$. Oxidative stress could disrupt RPE junction and barrier integrity, which may contribute to the pathogenesis of diseases related to RPE [20]. We found that high glucose milieu promotes ROS generation in a time and concentration-dependent manner. ROS is produced as the normal by-product of cellular metabolism. When produced in moderate amounts, ROS are thought to function as the signaling molecule in signal transduction pathways regulating cell proliferation, senescence, apoptosis, necrosis and autophagy [25]. Excess ROS could result in the accumulation of oxidized proteins in the ER. In general, the destroyed proteins are retrotranslocated to the cytoplasm and degraded by the ER-associated ubiquitine/proteasome degradation (ERAD) system. However, if the amount of unfolded and malfolded proteins exceeds the capacity of the ERAD system, the proteins start to aggregate in the ER and trigger ER stress [26, 27]. High glucose-induced ROS could activate ER stress signaling, and the interruption of ER stress signaling leads to the inhibition of autophagy in RPE. ER stress-regulated autophagy may 


\section{Cellular Physiology and Biochemistry}

Cell Physiol Biochem 2014;33:107-116

\begin{tabular}{l|l}
\hline DOI: $10.1159 / 000356654$ & (C) 2014 S. Karger AG, Basel
\end{tabular}

\begin{tabular}{l|l} 
Published online: January 17, 2014 & www.karger.com/cpb
\end{tabular}

Yao et al.: High Glucose Induces RPE Autophagy

play a critical role in the maintenance of normal physiological functions in RPE in response to excess energy crisis.

mTOR functions as a sensor of the cellular energy status, and its activity is inhibited upon energy stress. mTOR signaling is also reported to be involved in the regulation of autophagy activity [17]. mTOR could negatively regulate p73, a member of the p53 family of transcription factors that induces autophagy and autophagy-related genes [27]. Ulk1 is a serine/threonine kinase and the mammalian functional homolog of yeast Atg1. It has been shown to play a crucial role in the initiation step of autophagy. Under nutrient sufficiency, high mTOR activity prevents Ulk1 activation by phosphorylating Ulk1 Ser 757 and disrupting the interaction between Ulk1 and AMPK to affect the development of autophagy in HEK293 cells [28]. We also investigate the relationship between mTOR activation and RPE autophagy. We found that high glucose treatment activates mTOR signaling. However, the inhibition of mTOR signaling by rapamycin treatment has no effect on high glucose-mediated autophagy, suggesting that mTOR signaling is not a major signaling invovled in RPE autophagy.

In conclusion, the current report demonstrates that high levels of glucose could result in the induction of RPE autophagy. Mechanistically, autophagy induction is mediated, at least in part, by ROS-mediated ER stress signaling. To our knowledge, this is the first report of high glucose-induced autophagy in retinal cells. Further study will be needed to gain additional details about the regulatory mechanisms of high glucose-mediated autophagy in retina.

\section{Acknowledgements}

This work was generously supported by grants from the National Natural Science Foundation of China (Grant No. 81300241 to B.Y. and Grant No. 81371055 to Q.J.), grants from the National clinical key construction project [Grant No. (2012) 649 to Q.J.], and grants from the Medical Science and Technology Development Project Fund of Nanjing (Grant No. ZKX 12047 to Q.J., Grant No.YKK12207 to G.-F.C, and Grant No. YKK12208 to J.Y.).

\section{References}

1 Glick D, Barth S, Macleod KF: Autophagy: cellular and molecular mechanisms. J Pathol 2010;221:3-12.

2 Klionsky DJ, Emr SD: Autophagy as a regulated pathway of cellular degradation. Science 2000;290:17171721.

-3 Komatsu M, Waguri S, Ueno T, Iwata J, Murata S, Tanida I, Ezaki J, Mizushima N, Ohsumi Y, Uchiyama Y, Kominami E, Tanaka K, Chiba T: Impairment of starvation-induced and constitutive autophagy in Atg7deficient mice. J Cell Biol 2005;169:425-434.

4 Nixon RA: Autophagy in neurodegenerative disease: Friend, foe or turncoat? Trends Neurosci 2006;29:528535.

5 Tannous P, Zhu H, Johnstone JL, Shelton JM, Rajasekaran NS, Benjamin IJ, Nguyen L, Gerard RD, Levine B, Rothermel BA: Autophagy is an adaptive response in desmin-related cardiomyopathy. Proc Natl Acad Sci USA 2008;105:9745-9750.

6 Yang Z, Klionsky DJ: Mammalian autophagy: core molecular machinery and signaling regulation. Curr Opin Cell Biol 2010;22:124-131.

7 He F, Xia X, Wu XF, Yu XQ, Huang FX: Diabetic retinopathy in predicting diabetic nephropathy in patients with type 2 diabetes and renal disease: a meta-analysis. Diabetologia 2013;56:457-466.

$>8$ Hammes HP, Feng Y, Pfister F, Brownlee M: Diabetic retinopathy: targeting vasoregression. Diabetes 2011;60:9-16.

-9 Du M, Wu M, Fu D, Yang S, Chen J, Wilson K, Lyons TJ: Effects of modified LDL and HDL on retinal pigment epithelial cells: a role in diabetic retinopathy? Diabetologia 2013;56:2318-2328.

10 Xu HZ, Song Z, Fu S, Zhu M, Le YZ: RPE barrier breakdown in diabetic retinopathy: seeing is believing. J Ocul Biol Dis Infor 2011;4:83-92. 


\section{Cellular Physiology and Biochemistry}

Cell Physiol Biochem 2014;33:107-116

\begin{tabular}{l|l}
\hline DOI: $10.1159 / 000356654$ & (C) 2014 S. Karger AG, Basel
\end{tabular}

Yao et al.: High Glucose Induces RPE Autophagy

-11 Ablonczy Z, Dahrouj M, Tang PH, Liu Y, Sambamurti K, Marmorstein AD, Crosson CE: Human retinal pigment epithelium cells as functional models for the RPE in vivo. Invest Ophthalmol Vis Sci 2011;52:86148620.

12 Pitocco D, Tesauro M, Alessandro R, Ghirlanda G, Cardillo C: Oxidative stress in diabetes: implications for vascular and other complications. Int J Mol Sci 2013;14:21525-21550.

13 Jaganjac M, Tirosh O, Cohen G, Sasson S, Zarkovic N: Reactive aldehydes-second messengers of free radicals in diabetes mellitus. Free Radic Res 2013;1:S39-48.

14 Rosenfeldt MT, Ryan KM: The multiple roles of autophagy in cancer. Carcinogenesis 2011;32:955-963.

15 Mizushima N, Yoshimori T, Levine B: Methods in mammalian autophagy research. Cell 2010;140:313-326.

- 16 Klionsky DJ, Abdalla FC, Abeliovich H, Abraham RT, Acevedo-Arozena A, et al.: Guidelines for the use and interpretation of assays for monitoring autophagy. Autophagy 2012;8:445-544.

- 17 Jung CH, Ro SH, Cao J, Otto NM, Kim DH: mTOR regulation of autophagy. FEBS Lett 2010;584:1287-1295.

18 Scherz-Shouval R, Elazar Z: Regulati on of autophagy by ROS: physiology and pathology. Trends Biochem Sci 2011;36:30-38.

19 Orsi A, Fioriti L, Chiesa R, Sitia R: Conditions of endoplasmic reticulum stress favor the accumulation of cytosolic prion protein. J Biol Chem 2006;281:30431-30438.

-20 Simó R, Villarroel M, Corraliza L, Hernández C, Garcia-Ramírez M: The retinal pigment epithelium: something more than a constituent of the blood-retinal barrier-implications for the pathogenesis of diabetic retinopathy. J Biomed Biotechnol 2010;190724. doi: 10.1155/2010/190724.

-21 Shintani T, Klionsky DJ: Autophagy in health and disease: a double-edged sword. Science 2004;306:990995.

22 Ma T, Zhu J, Chen X, Zha D, Singhal PC, Ding G: High glucose induces autophagy in podocytes. Exp Cell Res 2013;319:779-789.

-23 Jung HS, Chung KW, Won Kim J, Kim J, Komatsu M, Tanaka K, Nguyen YH, Kang TM, Yoon KH, Kim JW, Jeong YT, Han MS, Lee MK, Kim KW, Shin J, Lee MS: Loss of autophagy diminishes pancreatic beta cell mass and function with resultant hyperglycemia. Cell Metab 2008;8:318-324.

24 Kobayashi S, Xu X, Chen K, Liang Q: Suppression of autophagy is protective in high glucose-induced cardiomyocyte injury. Autophagy 2012;84:577-592.

25 Sauer H, Wartenberg M, Hescheler J: Reactive o xygen species as intracellular messengers during cell growth and differentiation. Cell Physiol Biochem 2001;11:173-186.

-26 Rutkowski DT, Kaufman RJ: A trip to the ER: coping with stress. Trends Cell Biol 2004;14:20-28.

27 Rosenbluth JM, Pietenpol JA: mTOR regulates autophagy-associated genes downstream of p73. Autophagy 2009;5:114-116.

28 Kim J, Kundu M, Viollet B, Guan KL: AMPK and mTOR regulate autophagy through direct phosphorylation of Ulk1. Nat Cell Biol 2011;13:132-141. 


\section{Erratum}

***

In the article by Yao et al., entitled "Regulation of autophagy by high glucose in human retinal pigment epithelium" [Cell Physiol Biochem 2014;33:107-116. (Doi: 10.1159/000356654)] there is an error in Figure 4. The blots of p-eIF2 and p-eIF2 were inversely marked. The correct figure and the legend is reproduced correctly here. The authors apologize for the typographical oversight and any inconvenience caused. The results and conclusions of the article remain unchanged.

Fig. 4. High glucose induces autophagy through ROS-mediated ER stress signaling. (A) RPE cells were incubated with high glucose (30 $\mathrm{mM}$ ) for $12 \mathrm{~h}, 24 \mathrm{~h}$, or $48 \mathrm{~h}$, or incubated with the culture medium containing different concentrations of glucose (5 mM, $20 \mathrm{mM}, 25 \mathrm{mM}$, and $30 \mathrm{mM}$ ) for $48 \mathrm{~h}$. Intracellular ROS levels were detected using DCFDA dye. The group incubated with normal glucose (5 mM) was taken as the control group. The data was shown as relative change compared with the control group. “*” indicated significant difference compared with the control group. (B) RPE cells were incubated with the medium containing normal glucose (5 $\mathrm{mM}$, Ctrl), high glucose (30 mM), or mannitol (30 mM) for $48 \mathrm{~h}$. Western blots were conducted to detect the total amount and phosphorylated level of JNK protein. $\beta$-tubulin expression was detected as the loading control. A representative immunoblot is shown. (C) RPE cells were treated as shown Fig. 4B. Western blots were conducted to detect p-eIF2, eIF2, p-PERK, and PERK expression. The relative expression of each protein was expressed as the relative change compared with the control group. A representative immunoblot was shown along with the quantita-

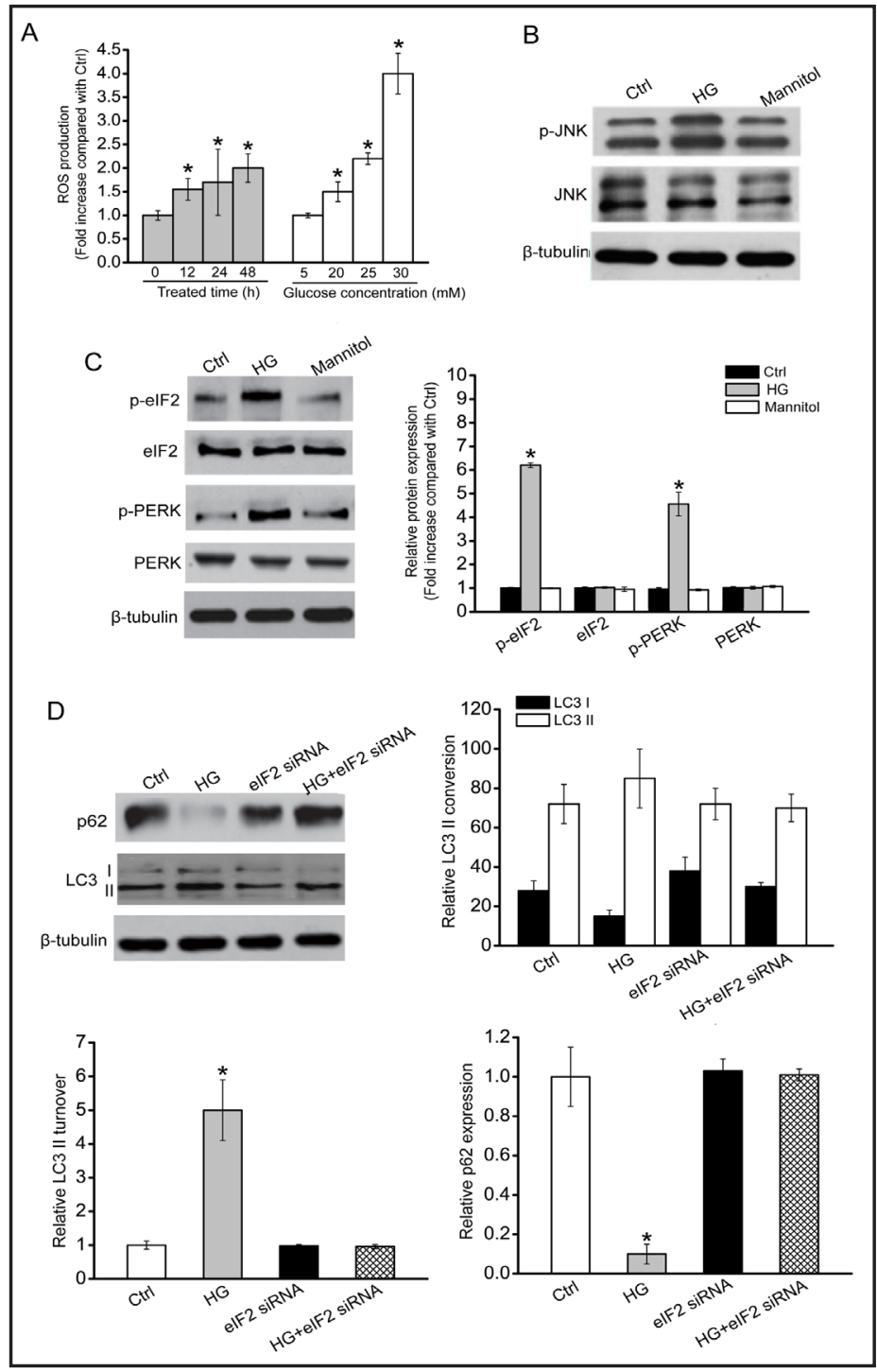
tive data from four separate blots. (D) RPE cells were transfected with eIF- $2 \alpha$ siRNA to silence ER stress signaling, and then incubated with the medium containing normal glucose ( $5 \mathrm{mM}$ ) or high glucose (30 $\mathrm{mM})$ for $48 \mathrm{~h}$. Western blots were conducted to detect LC3 and p62 expression. $\beta$-tubulin expression was detected as the loading control. The group treated with normal glucose was taken as the control group. LC3 or p62 expression was shown as relative change compared with the control group. A representative immunoblot was shown along with the quantitative data from four separate blots. 